\title{
Ultrasound imaging of immersed plates using high-order Lamb modes at their low attenuation frequency bands
}

\author{
Aline E. Takiy ${ }^{\mathrm{a}}$, Cláudio Kitano ${ }^{\mathrm{b}}$, Ricardo T. Higuti ${ }^{\mathrm{b}, *}$, Silvio C.G. Granja ${ }^{\mathrm{c}}$, Vander T. Prado ${ }^{\mathrm{d}}$, \\ Luis Elvira ${ }^{\mathrm{e}}$, Oscar Martínez-Graullera ${ }^{\mathrm{e}}$ \\ ${ }^{a}$ Centro Universitário Católico Salesiano Auxilium, Araçatuba, Brazil \\ ${ }^{\mathrm{b}}$ UNESP - Univ Estadual Paulista, Department of Electrical Engineering, Ilha Solteira, Brazil \\ ${ }^{\mathrm{c}}$ UNEMAT, Campus of Sinop, Brazil \\ ${ }^{\mathrm{d}}$ Department of Electrical Engineering, Universidade Tecnológica Federal do Paraná - UTFPR, Campus of Cornélio Procópio, Brazil \\ e ITEFI/CSIC - Consejo Superior de Investigaciones Científicas, Madrid, Spain
}

\section{A R T I C L E I N F O}

\section{Article history:}

Received 31 August 2016

Received in revised form 22 November 2016 Accepted 18 April 2017

Available online 26 April 2017

\section{Keywords:}

Lamb waves

Ultrasonic array

Imaging

Immersed plate

Attenuation

\begin{abstract}
A B S T R A C T
This paper focuses on the use of a Lamb wave-based methodology for ultrasound imaging of immersed plate structures. In these cases Lamb waves can be strongly attenuated due to leaky waves and viscous losses in the liquid, but there are low attenuation frequency bands that may be used for NDT applications. Experimental measurements were conducted to validate the existence of these low attenuation frequency bands, which were also theoretically predicted for some propagation modes, between the frequencies-thickness products of $0.5 \mathrm{MHz} \mathrm{mm}$ and $9.0 \mathrm{MHz} \mathrm{mm}$. Using a $5 \mathrm{MHz}$ linear-array and phased-array techniques, A1 and S1 modes are used to obtain images of an immersed aluminum plate with artificial defects. The signals are post-processed in order to select the desired propagation mode and to obtain an image with dynamic focusing in reception. While the A1 mode is strongly attenuated, the $\mathrm{S} 1$ mode, at $3.4 \mathrm{MHz} \mathrm{mm}$, can be used to detect and localize defects in the immersed plate.
\end{abstract}

(c) 2017 Elsevier Ltd. All rights reserved.

\section{Introduction}

Lamb waves are appealing for non-destructive testing (NDT) of plate and pipe structures, and structural health monitoring (SHM) in the aerospace and civil engineering industries, due to their characteristic to propagate relatively long distances (in dry conditions), allowing inspection of a significant large area without having to move the transducers [1-3]. Lamb wave techniques can be used to detect and monitor corrosion [4,5], cracks [6], and/or delaminations [7] in fiber and reinforced carbon composite materials. Other applications include oil, water and food pipes [8]; off-shore immersed structures [9]; or medical diagnostics [10]. Different modes can propagate simultaneously, each one with a particular frequency-velocity relationship, given by the dispersion curves. The possibility of multimode propagation provides more information in a measurement process when compared to ultrasonic systems based on monomode longitudinal or shear waves. On the other hand, the attenuation and dispersion characteristics must be taken into account for correct interpretation of results, since they may interfere with each other.

\footnotetext{
* Corresponding author.

E-mail address: tokio@dee.feis.unesp.br (R.T. Higuti).
} 
Most NDT applications with Lamb waves are focused on air-coupled or dry structures. For example, Zhu and Rose [11] study the generation of Lamb waves by using periodic linear arrays, in contact with a dry plate. When one or both sides of a plate are in contact with a liquid, there is an important mechanical coupling at the solid-liquid interfaces and strong attenuation can occur due to leaky waves and viscous losses in the fluid [12,13]. This attenuation significantly reduces the propagation range of the waves and limits its practical application in NDT.

When dealing with leaky waves, analytical approaches appropriate for gas-coupled plates and pipes are no longer valid, because fluid-solid coupling must be considered and, as a consequence, multidimensional systems arise. Different methods and numerical tools have been described in the literature to deal with this problem: the transfer-matrix method and the global-matrix method [14,15] the last being used in the software DISPERSE [16]; the semi-analytical finite element (SAFE) method [17-19], based on the coupling of harmonic function to a finite element method; the distributed point source method (DPSM) [20], based on the interface discretization to solve the coupling equations. The effect of a viscous loading layer on the attenuation of Lamb modes and shear horizontal modes were studied by Simoneti [21].

In spite of the acoustic coupling between the solid structure and the fluid, low attenuation frequency regions can be found for some guided modes. The attenuation drops when the out-of-plane oscillations at the solid-fluid interfaces tend to zero. In the case of cylindrical structures, these bands of minimal attenuation were investigated by Pavlakovic et al. [22], and can also be appreciated in the attenuation vs. frequency plots of many other works [23-25]. In plates, such bands have been studied, for example, by Zernov et al. [9] and Sharma and Mukherjee [26], although these attenuation plots are not shown as often as the sound velocity dispersion curves.

A variety of emitter/receiver configurations and propagation modes are reported in the literature when considering the inspection of immersed structures. Leinov et al. [25] analyzed the propagation through buried pipes to detect corrosion using a pulse-echo configuration, where the wave was excited by a ring of transducers around the pipes; Djili [27] analyzed immersed copper tubes using a similar pulse-echo arrangement, but exciting the tube with a single transducer; Mijarez et al. [28] used a pitch-catch configuration with a pair of transducers for SHM of sub-sea tubular structures. The TimeReversal technique [29] has also been used in the detection of cracks in a hollow cylinder immersed in water [30].

Chen et al. [5] used the A0 mode for analyzing liquid layers in contact with the plate using two immersion transducers operating in pitch-catch mode. Rizzo et al. [31], used a laser to excite the A0, S0 and quasi-Scholte modes and two immersion transducers were used for reception. In [32], a similar technique was used to detect Leaky Lamb waves by using an array of ultrasonic receivers. Immersed fiber reinforced carbon plates were analyzed by Chimenti and Martin [33] using leaky waves, but performing C-scans with a pair of immersion transducers. Recently, Sharma and Mukherjee [26] analyzed the mode propagation and defect detection in immersed plates exciting S0, A1 and S1 modes with a pair of immersed transducers. Zhang et al. [34] used a single transducer in a pulse-echo configuration with varying angle to detect cracks in immersed plates by means of lower order Lamb waves. Propagation of guided waves in plate structures was used to characterize fluids in contact with them [35], as well as in pipes [23].

This work is motivated by the industrial interest on NDT tools for the analysis of immersed structures. A linear array can be directly coupled to the structure under inspection, or by using wedges of appropriate geometries to excite the desired propagation modes. The use of Lamb wave arrays reduce the scanning requirements, as fast images of a given area can be obtained by placing the array at a fixed position. Special attention is given to mode selection and bandwidth limitation imposed by the low attenuation bands, as well as by employing an experimental inspection system with no moving parts. Such moving parts would not be practical in some applications, for example those involving difficult access to the structure. Depending on the desired range, the array can be moved, but even so the mechanical scanning requirements would be reduced in comparison with B- or C-Scan techniques, for example, which have been described in several works.

The underlying theory and mathematical models involving the propagation of Lamb waves in immersed plates are described in Section 2, along with experimental verifications of the low attenuation frequency bands. The model and associated theoretical curves are employed in Section 3 to properly select frequency bands and modes to propagate waves in immersed plates for NDT purposes. Low and high attenuation Lamb modes are excited by a linear array coupled to an aluminum plate with artificial defects. After signal acquisition and post-processing, an image of the structure is obtained for both dry and immersed conditions, showing the application of the proposed methodology. To the authors' knowledge, it is the first time that phased array technology combined with Lamb waves is used for imaging defects in immersed plate structures. Practical issues related to the use of this technology to NDT are discussed in Section 4.

\section{Dispersion and attenuation curves in immersed plates}

\subsection{Theoretical description}

Consider a $2 d$-thick isotropic solid plate immersed in a fluid, as illustrated in Fig. 1. The structure is unlimited along the $x$ and $y$ axes and wave propagation occurs in the $x$ direction. A geometrical discontinuity (plate-fluid) occurs only in the $z$ direction.

For fluid modeling, the equation of motion for a Newtonian viscoelastic fluid can be described by the Navier-Stokes equation [36], where shear and bulk viscosity coefficients were considered in the model. The shear viscosity of the liquid is 


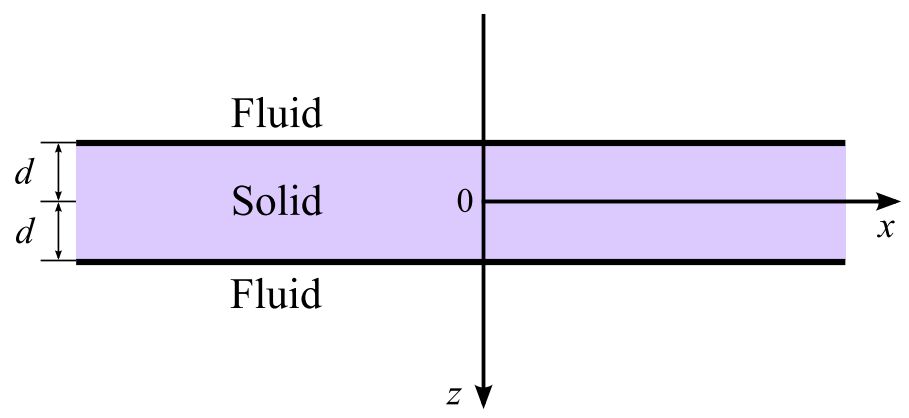

Fig. 1. Plate and fluid geometry for Lamb wave analysis.

responsible for a transversal stress coupling with the solid structure. On the other hand, both viscosity coefficients play a role as the imaginary part of the longitudinal wavenumber in the liquid, which is related to wave attenuation.

The dispersion characteristics for an immersed plate in a viscous fluid can be found by formulating particle velocity and stress components of the fluid and solid at the interfaces, yielding a set of eight equations, four corresponding to each side of the plate [15]. The symmetry obtained when the fluids on both sides of the plate are the same can be used to separate the problem in two homogeneous systems of four equations. The solutions of both $4 \times 4$ systems provide the symmetric and antisymmetric modes. The dispersion characteristic is obtained from (1) for the symmetric modes, and for the antisymmetric modes from (2), where $A, B, \ldots$, and $G$ are constants which depend on the excitation, and $j=\sqrt{-1}$.

$$
\begin{gathered}
{\left[\begin{array}{cccc}
\left(k^{2}+s^{2}\right) \cosh q d & 2 j k s \cosh s d & -r\left(k^{2}+\gamma_{2}^{2}\right) & -2 j r k \gamma_{2} \\
2 j k q \sinh q d & -\left(k^{2}+s^{2}\right) \sinh s d & -2 j r k \gamma_{1} & r\left(k^{2}+\gamma_{2}^{2}\right) \\
q \sinh q d & j k \sinh s d & -\gamma_{1} & -j k \\
j k \cosh q d & -s \cosh s d & -j k & \gamma_{2}
\end{array}\right]\left[\begin{array}{l}
A \\
D \\
E \\
G
\end{array}\right]=0,} \\
{\left[\begin{array}{cccc}
\left(k^{2}+s^{2}\right) \sinh q d & 2 j k s \sinh s d & -r\left(k^{2}+\gamma_{2}^{2}\right) & -2 j r k \gamma_{2} \\
2 j k q \cosh q d & -\left(k^{2}+s^{2}\right) \cosh s d & -2 j r k \gamma_{1} & r\left(k^{2}+\gamma_{2}^{2}\right) \\
q \cosh q d & j k \cosh s d & -\gamma_{1} & -j k \\
j k \sinh q d & -s \sinh s d & -j k & \gamma_{2}
\end{array}\right]\left[\begin{array}{l}
B \\
C \\
E \\
G
\end{array}\right]=0,}
\end{gathered}
$$

For the solid, $\rho_{s}$ is the mass bulk density; $k$ is the wavenumber of the guided mode; $q^{2}=k^{2}-k_{l s}^{2} ;$ and $s^{2}=k^{2}-k_{t s}^{2}$, where $k_{l s}$ and $k_{t s}$ are the longitudinal and transversal wavenumbers, respectively, and

$$
\begin{aligned}
& k_{l s}^{2}=\frac{\omega^{2}}{c_{l s}^{2}}, \\
& k_{t s}^{2}=\frac{\omega^{2}}{c_{t s}^{2}},
\end{aligned}
$$

where $\omega$ is the angular frequency, and $c_{t s}$ and $c_{l s}$ are the transversal and longitudinal phase velocities in the solid, respectively.

For the fluid, $\eta$ and $\zeta$ are the shear and second (volume) viscosity coefficients, respectively. $\gamma_{1}^{2}=k^{2}-k_{l f}^{2}$ and $\gamma_{2}^{2}=k^{2}-k_{t f}^{2}$, where $k_{l f}$ and $k_{t f}$ are the longitudinal and transversal wavenumbers for the fluid, respectively, and

$$
\begin{aligned}
& k_{\mathrm{tf}}^{2}=\frac{j \omega \rho_{f}}{\eta}, \\
& k_{\text {lf }}^{2}=\left[\frac{1}{k_{\text {fluid }}^{2}}+\frac{1}{j \omega \rho_{f}}\left(\zeta+\frac{4}{3} \eta\right)\right]^{-1},
\end{aligned}
$$

where $\rho_{f}$ is the mass bulk density of the fluid; $k_{\text {fluid }}=\omega / c_{l f}$ is the wavenumber (bulk); and $c_{l f}$ is the longitudinal phase velocity in the fluid. The ratio $r$ is defined as $r=-j \eta k_{t s}^{2} / \rho_{s} \omega$. This model provides similar expressions to those obtained using the bulk elastic modulus for the fluid employed by Nayfeh and Nagy [15], when the second viscosity is disregarded (Stokes model). 
Table 1

Material properties.

\begin{tabular}{lcc}
\hline Property & Water & Aluminum \\
\hline Density $\left(\mathrm{kg} / \mathrm{m}^{3}\right)$ & 1000 & 2700 \\
Longitudinal velocity $(\mathrm{m} / \mathrm{s})$ & 1500 & 6400 \\
Shear velocity $(\mathrm{m} / \mathrm{s})$ & - & 3000 \\
Shear viscosity (Pa s) & 0.001 & - \\
Bulk viscosity (Pa s) & 0.0028 & - \\
\hline
\end{tabular}

Zero determinants of the homogenous systems (1) and (2) are necessary conditions for a non-trivial solution. The solutions for $k$ in (1) and (2) are complex and provide information about phase velocity $(c)$, and attenuation $(\alpha)$, of the guided modes,

$$
k=\frac{\omega}{c}+j \alpha .
$$

From the dispersion curve ( $c$ versus $\omega$ ) the group velocities are (following [37])

$$
c_{g}=\left(\frac{1}{c}-\frac{\omega}{c^{2}} \frac{d c}{d \omega}\right)^{-1}
$$

The dispersion equations were solved for an aluminum plate immersed in water. Plate and fluid material properties used in these simulations are shown in Table 1 . The corresponding phase velocity and attenuation as a function of frequencythickness product are presented in Fig. 2, in solid lines. The symbols show experimental results that will be described later.

As the frequency-thickness product increases, the velocity of the fundamental modes approaches the Rayleigh velocity in an immersed plate (Fig. 2a). These propagation velocity curves are very little influenced by the fluid (gas or liquid) surrounding the aluminum plate.

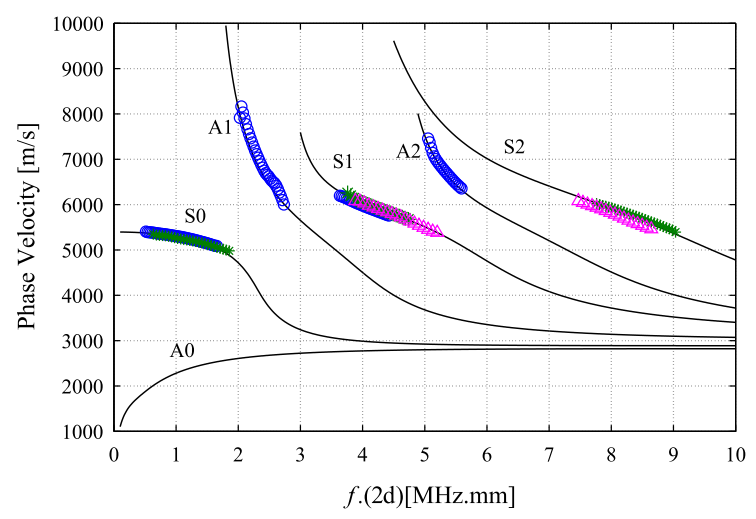

(a)

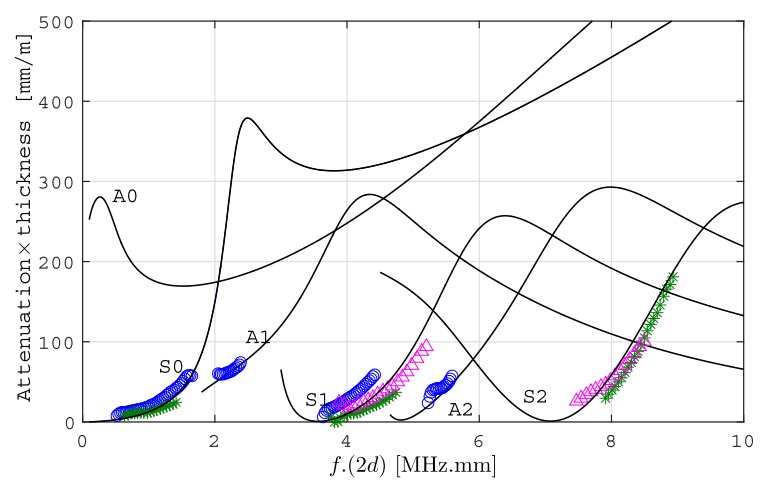

(b)

Fig. 2. (a) Dispersion curves and (b) attenuation curves. Simulated (solid lines) and experimental results were obtained for some propagation modes (blue circle for $1 \mathrm{~mm}$-thick plate; green plus for $2 \mathrm{~mm}$-thick plate and magenta triangle for 3-mm thick plate). (For interpretation of the references to colour in this figure legend, the reader is referred to the web version of this article.) 


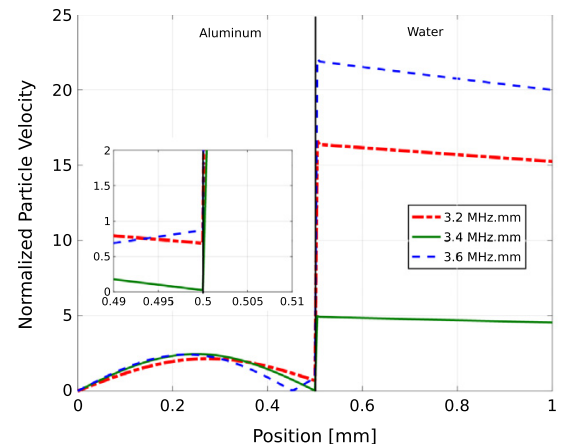

(a)

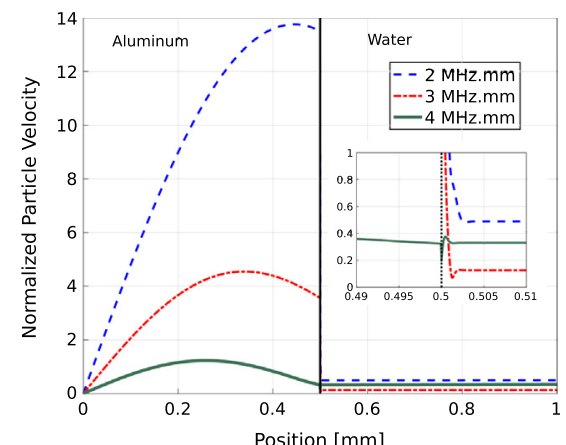

(b)

Fig. 3. Out-of-plane vibration velocities: (a) S1 mode around the attenuation minimum at $3.4 \mathrm{MHz}$. (b) A1 mode at 2, 3, and $4 \mathrm{MHz}$.

On the other hand, liquid loading can result in high attenuation due to leaky waves, and the attenuation characteristic (Fig. 2b) is very dependent on fluid properties. Despite the large attenuation, most modes show a band where the attenuation approaches zero. These attenuation minima can also be seen in [9] for the S1 and A2 modes. Similar relative minima can be found for higher order symmetric and antisymmetric modes. These minima are related to vanishing out-of-plane particle velocities at the plate surfaces. The vibrational profiles at these frequencies decrease the acoustic energy coupling from plate to fluid. In such cases, only in-plane vibration velocities exist at the interface, which results in some wave attenuation due to viscous losses but, ideally, no leaky waves.

As an example, the out-of-plane particle velocity profiles of the S1 mode for three frequencies around the minimum attenuation are shown in Fig. 3a. Out-of-plane vibration velocity vanishes at the plate surface only at $3.4 \mathrm{MHz}$ mm, which corresponds to the minimum attenuation point of operation of the S1 mode in Fig. 2. In the inset, a close view of the vibration distribution at the solid-liquid boundary confirms the zero and shows typical profiles of the viscous layer in the fluid phase. These attenuation minima allows the propagation of guided waves in immersed plates for structural evaluation purposes, for example by imaging an area of interest, as discussed in Section 3. On the other side, for the A1 mode around 3 MHz the outof-plane particle velocity (Fig. 2b) does not vanish at the plate surface, and there is no local minimum of attenuation. This non-zero particle velocity improves the wave coupling between the plate and the liquid, which results in a higher attenuation for this antisymmetric mode (Fig. 2b).

\subsection{Experimental verification of the low attenuation bands}

The existence of the low attenuation frequency bands of Lamb modes in an aluminum plate immersed in water is verified by using the experimental setup shown in Fig. 4. Lamb waves were generated by longitudinal wave transducers coupled to acrylic wedges operating in pitch-catch mode. The wedges were attached to a rail to ensure alignment between emitter and receiver. The transmitter ( $5 \mathrm{MHz}$ central frequency) was excited either with a short pulse (Olympus 5077PR Pulser-Receiver) or with a Gaussian envelope tone burst, obtained from a function generator (Tektronix AFG3101, 14 bits resolution) and a

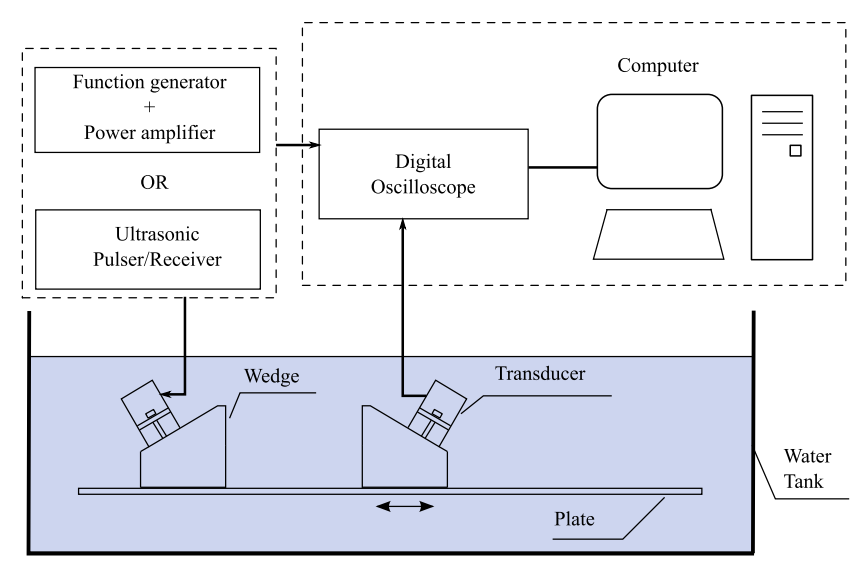

Fig. 4. Experimental setup for measurement of dispersion and attenuation curves. 
power amplifier (E\&I 240L, $40 \mathrm{~W}$ ). The received echoes were digitized by an oscilloscope (Agilent MSO7014B, 10 bits resolution in average mode) and transferred to a computer for post-processing.

The phase velocity of the guided mode coupled to the plate was obtained from the Snell-Descartes law: $c=c_{w} / \sin \theta_{w}$, where $c_{w}$ is the longitudinal propagation velocity in the wedge, and $\theta_{w}$ is the incidence angle relative to the plate normal. For example, using an acrylic wedge $\left(c_{w}=2700 \mathrm{~m} / \mathrm{s}\right)$ and $\theta_{w}=30^{\circ}$ and $25^{\circ}$, phase velocities of 5400 and $6388 \mathrm{~m} / \mathrm{s}$ were obtained, respectively. Since there is beam spreading and a finite bandwidth of operation, there is also a range of angles, and consequently a range of velocities, that can be coupled to the plate. For example, at approximately $3.5 \mathrm{MHz}$, using the $25^{\circ}$ wedge, A1 and S1 modes could be coupled. Aluminum plates with 1,2 , and 3 mm thicknesses were used to obtain the frequency-thickness product range used in the simulations.

\subsubsection{Mode propagation}

To compare the effects of mode attenuations in air-plate-air and water-plate-water configurations, the transducers were coupled to a $1-\mathrm{mm}$ thickness plate by a $25^{\circ}$ wedge and a pulser-receiver was used to excite the transmitter with a wideband pulse. Signals were obtained for the $1 \mathrm{~mm}$-thick aluminum plate in air and then immersed in water.

Received signals and the respective spectrograms (time-frequency, in gray scale) for the plate in air are shown in Fig. 5. The theoretical arrival times (group delays) obtained from the group velocity of each Lamb mode are also shown (solid lines) superposed to the experimental results. These group delays were obtained from $c_{g}=L / \Delta t$, where $\Delta t$ is the time of flight of each Lamb mode, i.e., the group delay, and $L$ is the distance between transducers. Except for the A0 mode, which cannot be

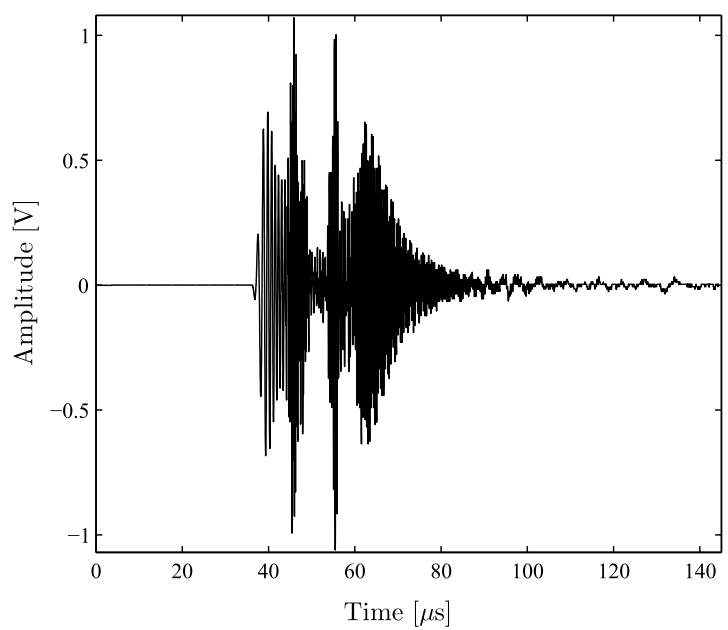

(a)

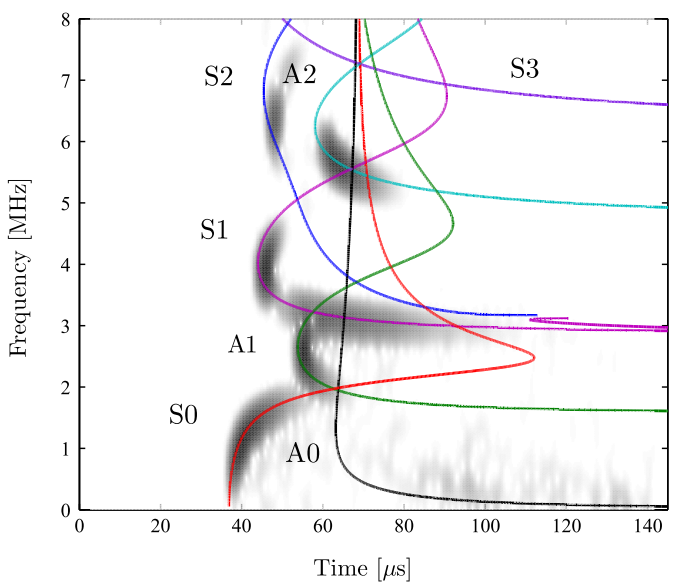

(b)

Fig. 5. Wave propagation in an aluminum plate in air, using wideband excitation: (a) time-domain received signal, and (b) spectrogram of the received signal (gray scale) superposed on the simulated dispersion curves with respect to group velocity (solid lines). 
coupled due to its low phase velocity, five modes, up to S2 and A2, can be observed when using these transducers and wedges.

The echoes and spectrogram for the plate immersed in water are shown in Fig. 6. The antisymmetric modes are strongly attenuated due to the leaky waves, while the symmetric modes are somewhat less attenuated.

\subsubsection{Measurement of mode attenuation}

The distance $L$ between the emitter and receiver wedges was varied from $15 \mathrm{~mm}$ to $85 \mathrm{~mm}$, with $1 \mathrm{~mm}$ step. Time traces were acquired for each distance, and the spectrum $S_{m}(\omega)$ of each signal was obtained, which is modelled as:

$$
S_{m}(\omega)=\widetilde{A} e^{-\alpha(\omega) x_{m}} e^{j \phi(\omega)}
$$

where $A(\omega)=\widetilde{A} e^{-\alpha(\omega) x_{m}}$ is the wave magnitude, $\alpha(\omega)$ is the attenuation, $\phi(\omega)$ is the phase of the wave with angular frequency $\omega$, and $x_{m}$ is the distance between the wedges. Linear fitting of $\ln (A) \times x$ and $\phi \times x$ were performed, allowing the calculation of attenuations and phase velocities, respectively.

The experimental results, obtained with 1, 2, and $3 \mathrm{~mm}$ thick plates and different frequencies, are superposed to the simulated dispersion and attenuation curves in Fig. 2 as $\bigcirc,+$, and $\triangle$ symbols, respectively.

There is good agreement between the theoretical and experimental velocity dispersion curves. The attenuation curves clearly have the same behavior and the lower attenuation regions can be found at approximately $1 \mathrm{MHz}$ mm (SO mode), 2.5 $\mathrm{MHz} \mathrm{mm}$ (A1 mode), 3.5 MHz mm (S1 mode), $5.5 \mathrm{MHz} \mathrm{mm}$ (A2 mode), and $8 \mathrm{MHz} \mathrm{mm}$ (S2 mode).

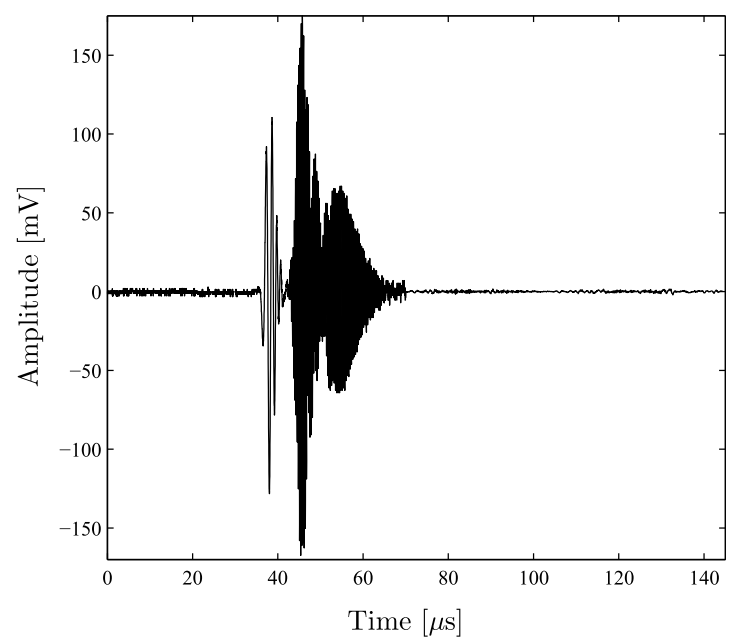

(a)

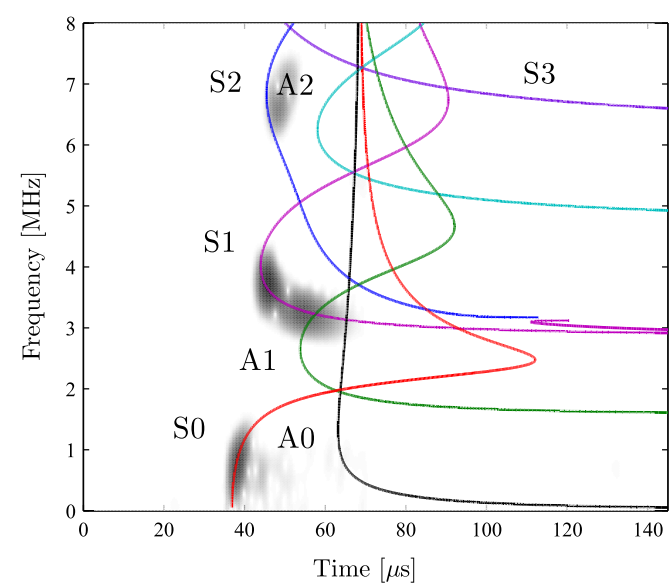

(b)

Fig. 6. Wave propagation in an aluminum plate immersed in water using wideband excitation: (a) time-domain received signal, and (b) spectrogram of the received signal (gray scale) superposed on the simulated dispersion curves with respect to group velocity (solid lines). 


\section{Ultrasonic imaging of immersed plates}

A $5 \mathrm{MHz}$ linear array with 128 elements, $0.65 \mathrm{~mm}$ pitch, and 73\% bandwidth (Imasonic, Besançon, France) was coupled to a $25^{\circ}$ incidence angle acrylic wedge (longitudinal velocity of $2700 \mathrm{~m} / \mathrm{s}$ ), which generated the A1 and $\mathrm{S} 1$ modes at approximately $3.5 \mathrm{MHz}$ in a $1 \mathrm{~mm}$-thick aluminum plate. An array system (SITAU, 32:128, DASEL S. L., Spain) was used to control the array, which was excited with wideband square programmable pulses ( $-90 \mathrm{~V}$ peak, 12 bits acquisition, $40 \mathrm{MHz}$ sampling rate).

The aluminum plate has dimensions of $200 \mathrm{~mm} \times 300 \mathrm{~mm}$ and the array was positioned at one of the borders of the plate, as shown in Fig. 7. Five artificial defects (drilled holes) were produced with 5 and $1.5 \mathrm{~mm}$ diameters (defects $a$ to $e$ ). The approximate area considered for imaging is delimited by the dashed line in Fig. 7.

A fixed aperture of 32 elements operating in phased-array mode was used, with fixed focus in emission, at $150 \mathrm{~mm}$. The array system was not used to process the received signals, but these were transferred to the computer for post-processing. Different from the excitation and processing used in the previous section for characterizing the low attenuation bands, the array system excites the transducer elements with a one-cycle square wave pulse. This wideband excitation generates the propagation of more than one mode simultaneously, in general at different frequencies and with different propagation velocities. Interpolation and dynamic-focusing in reception were implemented in the computer, using Matlab.
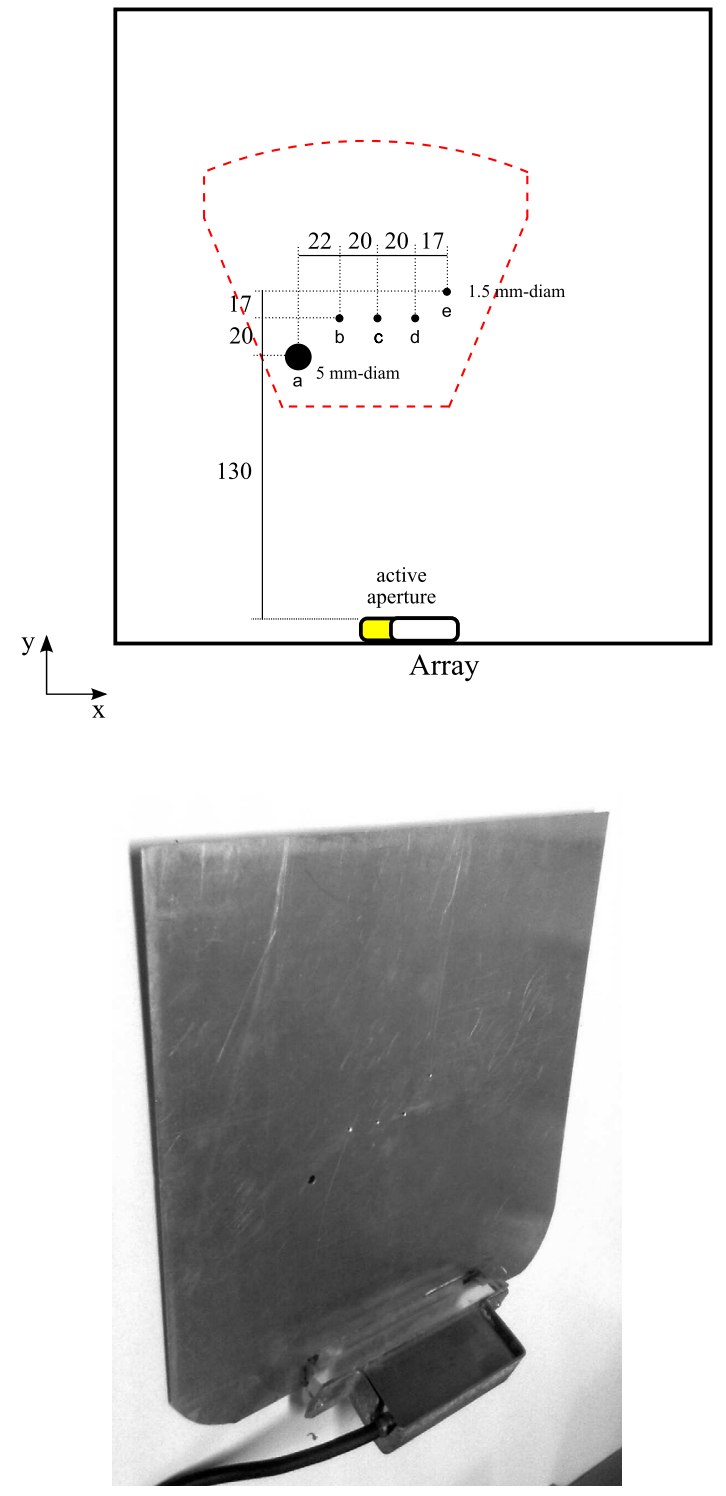

Fig. 7. Aluminum plate with artificial defects. Top: schematic, with dimensions in $\mathrm{mm}$. The dashed line limits the approximate area considered for imaging. Bottom: photo of the linear array and wedge attached to the plate. 
The ultrasonic images were obtained by using the group velocities for beamforming. The A1 and S1 modes show high dispersion, and phase and group velocities are very different. For example, for the $S 1$ mode at $3.4 \mathrm{MHz}$, the phase velocity is around $6500 \mathrm{~m} / \mathrm{s}$, whereas the group velocity is $3858 \mathrm{~m} / \mathrm{s}$. In post-processing and prior to beamforming, the wideband echoes are bandpass filtered at the frequencies of interest (bandwidth of $300 \mathrm{kHz}$ ), resulting in narrowband wave packets that are used in the imaging algorithm. The group velocity is defined in terms of a narrowband signal [38], and consequently for the signals considered in this work the group velocity is more adequate to characterize the pulse propagation than the phase velocity. In the case of dispersive signals, there are techniques for dispersion compensation [39] that have been employed by the authors in a previous work [40], where a high-dispersive A1 mode was used. In the noncompensated image, the defects appeared at wrong positions, even using a correct group velocity. Nevertheless, the narrowband operation used in the present work helps to minimise the effects of dispersion, and the additional amount of calculus does not improve the images considerably, consequently no dispersion compensation was applied in this work.

In order to compare the images and the attenuation influence, the plate was first inspected in air and then in water. First the frequency response of the system is experimentally obtained, in order to select adequate frequencies for operation in air and in water. These frequencies are then used to produce images that highlight the effects of attenuation of the guided waves.

\subsection{Experimental results}

Using the $25^{\circ}$ wedge and the $5 \mathrm{MHz}$ center frequency array, A1 and S1 modes were selected to highlight the attenuation effects, because the $\mathrm{A} 1$ mode has high attenuation in water, while the $\mathrm{S} 1$ mode has a lower attenuation frequency band that can be explored for imaging.

The frequency response is an important characteristic in mode selection, and depends on the array-wedge-plate system. The frequency responses of both modes were obtained by using the signal transmitted by element 1 of the array, which travels until a known reflector (defect $a$ ) and is received by element 32 . This known reflector could be also a small rod bonded to the plate, for example, and not necessarily a through-hole.

The results are shown in Fig. 8, for the plate in air (red and blue) and immersed in water (black and green). From the knowledge of the velocity dispersion curves, the wideband signals were windowed to select the A1 and S1 modes and then narrowband filtered at the various frequencies, from 2.5 to $4.75 \mathrm{MHz}$. The results were then normalized by the peak of the A1 mode in air, at $3 \mathrm{MHz}$. The $\mathrm{S} 1$ mode in air shows a lower amplitude than the A1 mode in air up to $3.6 \mathrm{MHz}$. For higher frequencies, the A1 mode magnitude decreases and the S1 mode magnitude increases up to $4.35 \mathrm{MHz}$.

When the plate is immersed in water, there is a strong attenuation of the A1 mode, by more than $50 \mathrm{~dB}$, while the $\mathrm{S} 1$ mode, at $3.4 \mathrm{MHz}$, is substantially less attenuated, by around $10 \mathrm{~dB}$. The effect of attenuation can be clearly observed for the $\mathrm{S} 1$ mode, where the minimum attenuation is around $3.4 \mathrm{MHz}$, as predicted from Fig. $2 \mathrm{~b}$.

Using this information, frequency bands and the corresponding modes were selected for image beamforming in air and in water to highlight the attenuation effect: A1 mode in air and in water at $3 \mathrm{MHz}$ (group velocity $c_{g}=3390 \mathrm{~m} / \mathrm{s}$ ); $\mathrm{S} 1$ mode in air at $4.35 \mathrm{MHz}\left(c_{g}=3997 \mathrm{~m} / \mathrm{s}\right)$ and $\mathrm{S} 1$ mode in water at $3.4 \mathrm{MHz}\left(c_{g}=3858 \mathrm{~m} / \mathrm{s}\right)$. The group velocities were obtained from (8).

The image of the plate in air, for both modes, is shown in Fig. 9, normalized by the A1 response. This image was obtained by using the propagation velocity of the $\mathrm{S} 1$ mode at $4.35 \mathrm{MHz}\left(c_{g}=3997 \mathrm{~m} / \mathrm{s}\right)$. The defects detected by the $\mathrm{S} 1$ mode $\left(a_{S 1}, b_{S 1}, \ldots\right)$ appear at their correct positions, approximately $y=15 \mathrm{~cm}$ for defects $b, c$ and $d$ (see solid line), for example. The defects that appear with higher intensities (around $y=17.7 \mathrm{~cm}$, or defects $b_{A 1}, c_{A 1}$ and $d_{A 1}$, see dashed line) are resulting from the A1 mode at $3 \mathrm{MHz}$, whose beamforming was made using the propagation velocity of the S1 mode, and, consequently, these defects do not appear at their correct positions. The difference in magnitude is expected because of the higher response of the $\mathrm{A} 1$ mode at $3 \mathrm{MHz}$ (see Fig. 8), and, in this dry condition, the defects are detected by both modes.

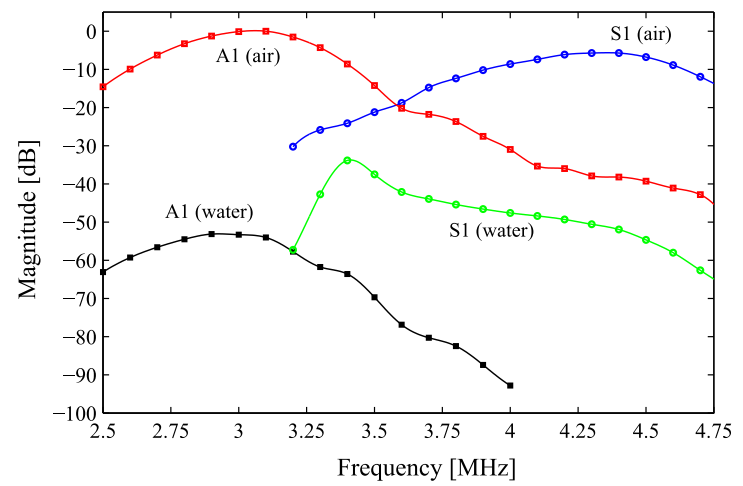

Fig. 8. Frequency responses of the A1 mode (squares) and S1 mode (circles). Propagation with plate in air (red and blue) and in water (black and green). (For interpretation of the references to colour in this figure legend, the reader is referred to the web version of this article.) 


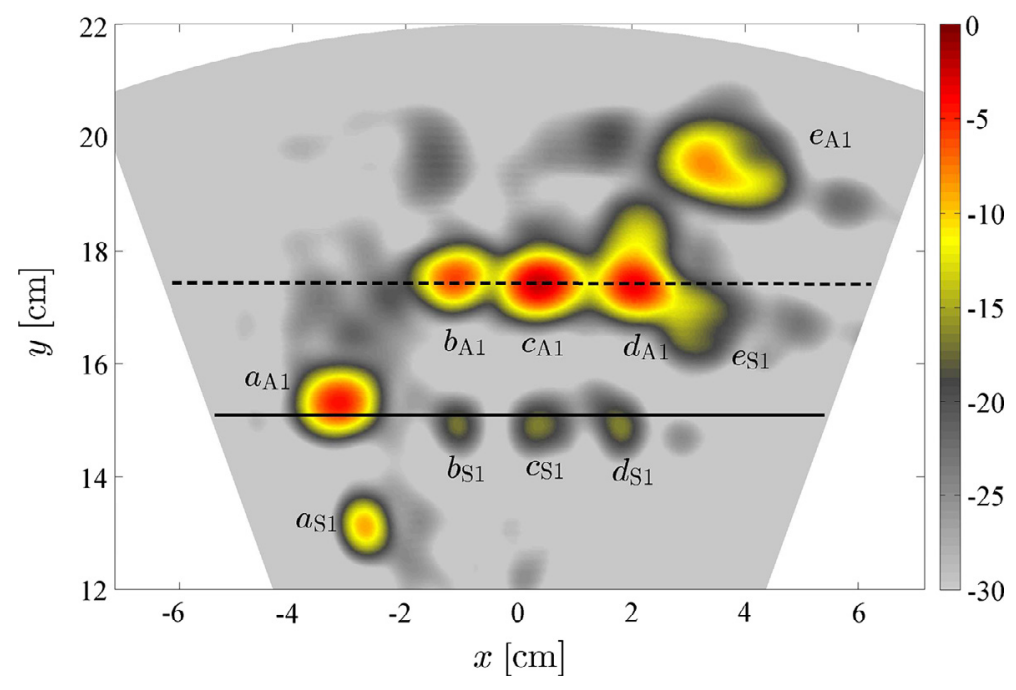

Fig. 9. Image of the plate in air, using the $\mathrm{S} 1$ mode propagation velocity of $3997 \mathrm{~m} / \mathrm{s}$.

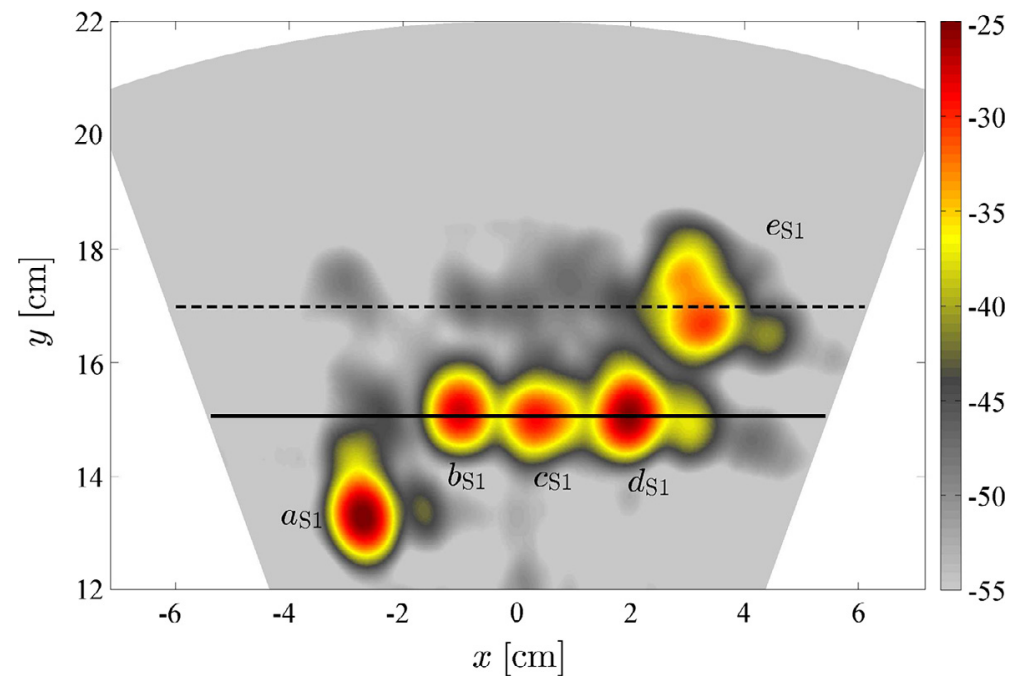

Fig. 10. Image of the plate in water, using the $\mathrm{S} 1$ mode propagation velocity of $3858 \mathrm{~m} / \mathrm{s}$.

Fig. 10 shows the image of the plate when it is immersed in water, using a different dynamic range. This image was obtained with the propagation velocity of the $\mathrm{S} 1$ mode at $3.4 \mathrm{MHz}$. The A1 mode at $3 \mathrm{MHz}$ is strongly attenuated because of the leaky waves, preventing detection of defects. On the other hand, the defects can still be visualized using the lower attenuation $\mathrm{S} 1$ mode at $3.4 \mathrm{MHz}$.

To compare the relative amplitudes between the images in air and in water, the intensity of the pixels at the center of each defect from Figs. 9 and 10 are shown in Table 2. The S1 mode suffered a maximum attenuation of $19.6 \mathrm{~dB}$, for defect $a$, while for the A1 mode in water it was not possible to obtain significant values (asterisks), due to the strong attenuation effect, as predicted by the frequency response.

Table 2

Image values (in $\mathrm{dB}$ ) at defects positions, obtained from Figs. 9 and 10.

\begin{tabular}{lcccccc}
\hline Mode & Medium/defect & $a$ & $b$ & $c$ & $d$ & -1.3 \\
\hline A1 & Air & -1.2 & -3.8 & 0 & $*$ & $*$ \\
& Water & $*$ & $*$ & $*$ & -14.4 & -14.3 \\
S1 & Air & -6.7 & -14.7 & -29.3 & -27.2 & -13.1 \\
& Water & -26.3 & -29.1 & & -32.3 \\
\hline
\end{tabular}


In the beamformed image, there is contribution of all array elements, and this will include several propagation effects, such as mode coupling, reflection coefficients, radiation pattern and attenuation. Although the dispersion and attenuation curves, as well as the frequency response, were obtained from only a particular condition (a pair of transmitter-receiver), this procedure, of analyzing the velocity and attenuation curves as a function of frequency, is extremely important and effective to determine an optimized operation point for imaging purposes using Lamb waves. A useful aspect is the "mode cleaning" effect in the immersed plate, because modes with higher attenuation tend to disappear, and there are less artifacts produced by spurious modes in the final image.

\section{Conclusions}

The main contribution of this article is the establishment of a procedure for the characterization and selection of adequate Lamb modes to be used in image beamforming using a phased-array system and post-processing in immersed structures. Although there is still some attenuation involved when compared to the propagation in dry conditions, the linear array coupled to a wedge can be moved to some parts of the structure, resulting in less mechanical moving parts than a conventional B- or C-Scan technique, and can be used to help to locate defects positions. Signal processing was important in the problem analysis and image beamforming, in order to select the desired propagation modes and reach adequate image resolution.

The authors have not observed imaging of immersed plates using this methodology in the literature. These experimental results have a great interest, since it confirms that propagation of high frequency Lamb waves is also possible in immersed conditions by suitable choice of frequency band and mode. Moreover, under these circumstances the attenuation spectrum shows that only one Lamb mode presents a minimum attenuation value for a given frequency. Consequently, when the plate is immersed in a fluid, an increase in operating frequency does not give rise to modal interference as occurs in air-coupled plates.

\section{Acknowledgements}

The authors would like to thank the funding agencies: CAPES - Brazil, CNPq - Brazil (560825/2010-2) and FAPESP - Brazil (2014/08790-3); MINECO - Spain (PIB2010BZ-00570); and Professor Julio C. Adamowski, from USP - São Paulo University, for providing the $5 \mathrm{MHz}$ wideband transducers.

\section{References}

[1] J.L. Rose, Ultrasonic Waves in Solid Media, Cambridge University Press, UK, 1999.

[2] P.D. Wilcox, Omni-directional guided wave transducer arrays for the rapid inspection of large areas of plate structures, IEEE Trans. Ultrason., Ferroelectr., Freq. Control 50 (6) (2003) 699-709.

[3] K. Diamanti, C. Soutis, Structural health monitoring techniques for aircraft composite structures, Prog. Aerosp. Sci. 46 (8) (2010) $342-352$.

[4] V. Rathod, D.R. Mahapatra, Ultrasonic Lamb wave based monitoring of corrosion type of damage in plate using a circular array of piezoelectric transducers, NDT\&E Int. 44 (7) (2011) 628-636.

[5] J. Chen, Z. Su, L. Cheng, Identification of corrosion damage in submerged structures using fundamental anti-symmetric Lamb waves, Smart Mater. Struct. 19 (1) (2010) 1-12.

[6] X. Chen, J.E. Michaels, S.J. Lee, T.E. Michaels, Load-differential imaging for detection and localization of fatigue cracks using Lamb waves, NDT\&E Int. 51 (2012) 142-149.

[7] K. Tan, N. Guo, B. Wong, C. Tui, Comparison of Lamb waves and pulse echo in detection of near-surface defects in laminate plates, NDT\&E Int. 28 (4) (1995) 215-223.

[8] T.R. Hay, J.L. Rose, Fouling detection in the food industry using ultrasonic guided waves, Food Control 14 (7) (2003) $481-488$.

[9] V. Zernov, L. Fradkin, P. Mudge, Guided waves in a monopile of an offshore wind turbine, Ultrasonics 51 (1) (2011) $57-64$.

[10] K. Xu, D. Liu, D. Ta, B. Hu, W. Wang, Quantification of guided mode propagation in fractured long bones, Ultrasonics 54 (5) (2014) 1210-1218.

[11] W. Zhu, J.L. Rose, Lamb wave generation and reception with time-delay periodic linear arrays: a BEM simulation and experimental study, IEEE Trans. Ultrason., Ferroelectr., Freq. Control 46 (3) (1999) 654-664.

[12] A.H. Nayfeh, Wave Propagation in Layered Anisotropic Media: With Applications to Composites, North Holland, 1995.

[13] C.H. Yang, C. Shue, Guided waves propagating in a piezoelectric plate immersed in a conductive fluid, NDT\&E Int. 34 (3) (2001) $199-206$.

[14] M.J.S. Lowe, Matrix techniques for modeling ultrasonic waves in multilayered media, IEEE Trans. Ultrason., Ferroelectr., Freq. Control 42 (4) (1995) $525-542$.

[15] A.H. Nayfeh, P.B. Nagy, Excess attenuation of leaky Lamb waves due to viscous fluid loading, J. Acoust. Soc. Am. 101 (5) (1997) $2649-2658$.

[16] B. Pavlakovic, M. Lowe, D. Alleyne, P. Cawley, DISPERSE: a general purpose program for creating dispersion curves, Rev. Prog. Quant. Nondestruct. Eval. 16 (1997) 185-192.

[17] T. Hayashi, K. Kawashima, Z. Sun, J.L. Rose, Analysis of flexural mode focusing by a semianalytical finite element method, J. Acoust. Soc. Am. 113 (3) (2003) 1241-1248.

[18] T. Hayashi, W.-J. Song, J.L. Rose, Guided wave dispersion curves for a bar with an arbitrary cross-section, a rod and rail example, Ultrasonics 41 (3) (2003) 175-183.

[19] A. Marzani, E. Viola, I. Bartoli, F.L. di Scalea, P. Rizzo, A semi-analytical finite element formulation for modeling stress wave propagation in axisymmetric damped waveguides, J. Sound Vib. 318 (2008) 488-505.

[20] S. Banerjee, T. Kundu, Ultrasonic field modeling on plates immersed in fluid, Int. J. Solids Struct. 44 (2007) 6013-6029.

[21] F. Simonetti, Lamb wave propagation in elastic plates coated with viscoelastic materials, J. Acoust. Soc. Am. 115 (5) (2004) $2041-2053$.

[22] B. Pavlakovic, M. Lowe, P. Cawley, High-frequency low-loss ultrasonic modes in imbedded bars, J. Appl. Mech. 68 (2001) 67-75.

[23] F. Simonetti, P. Cawley, A guide wave technique for the characterization of highly attenuative viscoelastic materials, J. Acoust. Soc. Am. 114 (1) (2004) $158-165$.

[24] L. Elvira-Segura, Acoustic wave dispersion in a cylindrical elastic tube filled with a viscous liquid, Ultrasonics 37 (2000) $537-547$.

[25] E. Leinov, M. Lowe, P. Cawley, Investigation of guide wave propagation and attenuation in pipe buried in sand, J. Sound Vib. 347 (2015) $96-114$.

[26] S. Sharma, A. Mukherjee, Damage detection in submerged plates using ultrasonic guided waves, Sadhana 39 (5) (2014) $1009-1034$. 
[27] S. Djili, F. Benmeddour, E. Moulin, J. Assaad, F. Boubenider, Notch detection in copper tubes immersed in water by leaky compressional guided waves, NDT\&E Int. 54 (2013) 183-188.

[28] R. Mijarez, P. Gaydecki, M. Burdekin, Flood member detection for real-time structural health monitoring of sub-sea structures of offshore steel oilrigs, Smart Mater. Struct. 16 (2007) 1857-1869.

[29] R.K. Ing, M. Fink, Time-reversed Lamb waves, IEEE Trans. Ultrason., Ferroelectr, Freq. Control 45 (4) (1998) 1032-1043.

[30] E. Kerbrat, D. Clorennec, C. Prada, D. Royer, D. Cassereau, M. Fink, Detection of cracks in a thin air-filled hollow cylinder by application of the DoRT method to elastic components of the echo, Ultrasonics 40 (1-8) (2002) 715-720.

[31] P. Rizzo, J. Han, X. Ni, Structural health monitoring of immersed structures by means of guided ultrasonic waves, J. Intell. Mater. Syst. Struct. 21 (14) (2010) 1397-1407.

[32] E. Pistone, K. Li, P. Rizzo, Noncontact monitoring of immersed plates by means of laser-induced ultrasounds, Struct. Health Monitor. 12 (5-6) (2013) $549-565$.

[33] D. Chimenti, R.W. Martin, Nondestructive evaluation of composite laminates by leaky Lamb waves, Ultrasonics 29 (1) (1991) $13-21$.

[34] Y. Zhang, Y. Sidibé, G. Mazec, F. Leon, F. Druaux, D. Lefebvre, Detection of damages in underwater metal plate using acoustic inverse scattering and image processing methods, Appl. Acoust. 103A (2016) 110-121.

[35] F. Cegla, P. Cawley, M. Lowe, Material property measurement using the quasi-Scholte mode - a waveguide sensor, J. Acoust. Soc. Am. 117 (3) (2005) 1098-1107.

[36] L.D. Landau, E.M. Lifshitz, Fluid Mechanics, Pergamon Press, 1966.

[37] B. Auld, Acoustic Fields and Waves in Solids, vol. 1, R.E. Krieger, 1990.

[38] R.B. Carlson, Communication Systems: An Introduction to Signals and Noise in Electrical Communication, third ed., McGraw-Hill, Singapore, 1986.

[39] P.D. Wilcox, A rapid signal processing technique to remove the effect of dispersion from guided wave signals, IEEE Trans. Ultrason., Ferroelectr., Freq. Control 50 (4) (2003) 419-427.

[40] V. Prado, R.T. Higuti, C. Kitano, O. Martínez-Graullera, J.C. Adamowski, Lamb mode diversity imaging for non-destructive testing of plate-like structures, NDT \& E Int. 59 (2013) 86-95. 